\title{
La Escala EFECO para Valorar Funciones Ejecutivas en Formato de Auto- Reporte
}

\author{
EFECO Scale for Assessing Executive Functions in Self-Report Format
}

\author{
Carlos Ramos-Galarza ${ }^{1}$, Mónica Bolaños-Pasquel², Andrés García-Gómez ${ }^{3}$, Pedro Martínez- \\ Suárez ${ }^{4}$ y Janio Jadán-Guerrero ${ }^{2}$
}

\section{Resumen}

El cuestionario EFECO es un instrumento que permite valorar las funciones ejecutivas desde una perspectiva ecológica, ya que se basa en el reporte conductual de la vida diaria del sujeto. El objetivo de la investigación fue validar el cuestionario EFECO en un formato de auto-reporte. Participaron 250 adolescentes ecuatorianos entre 13 y 18 años de edad $\left(M_{\text {edad }}=16.23, D E=1.73\right)$. Se encontraron resultados significativos en consistencia interna $(\alpha=.64-.95)$, correlación entre ítems $(r=.32-.62)$, correlación entre sub-escalas $(r=.40-.76)$, análisis factorial confirmatorio de dos factores $\left(x^{2}=38.87, C F I=.98, R M S E A=.06\right.$ [.035-.094], SRMR=.027) y capacidad discriminante en monitorización, flexibilidad cognitiva, organización de materiales, iniciativa, memoria de trabajo y planificación de adolescentes con diferente rendimiento académico. Se discuten los resultados en relación a estudios previos y bajo la importancia de contar con un instrumento con validez ecológica para evaluar funciones ejecutivas.

Palabras clave: cuestionario EFECO en auto-reporte, evaluación neuropsicológica, funciones ejecutivas, neuropsicología

\begin{abstract}
EFECO questionnaire is an instrument that allows assessing executive functions from an ecological perspective because it is based on a day-by-day behavioral report of an individual. The aim was to validate EFECO questionnaire in a self-report format. Participants were 250 Ecuadorian adolescents aged between 13 and 18 years $\left(M_{\mathrm{age}}=16.23, S D=1.73\right)$. As results, there was found a significant internal consistency $(\alpha=.64-$ $.95)$, correlation among items $(\mathrm{r}=.32-.62)$, correlation among sub-scales $(\mathrm{r}=.40-.76)$, confirmatory factor analysis of two factors $\left(\mathrm{x}^{2}=38.87, \mathrm{CFI}=.98, \mathrm{RMSEA}=.065\right.$ [.035-.094], SRMR=.027) and discriminant capacity in monitoring, cognitive flexibility, organization of material, initiative, working memory and planning of adolescents with differences on academic performance. In the discussion part, results are presented in relation to previous findings and taking on account the importance of having an instrument with an ecological validity to assess executive functions.
\end{abstract}

Keywords: EFECO questionnaire self-reported, executive functions, neuropsychological assessment, neuropsychologyKeywords

\footnotetext{
${ }^{1}$ Facultad de Psicología. Pontificia Universidad Católica del Ecuador, Ecuador.

${ }^{2}$ Centro de Investigación MIST. Universidad Tecnológica Indoamérica de Ecuador.

${ }^{3}$ Facultad de Psicología. Universidad de Extremadura, España.

${ }^{4}$ Facultad de Psicología. Universidad Católica de Cuenca, Ecuador.

Correspondencia: Carlos Ramos-Galarza PhD, Profesor Principal de la Facultad de Psicología, Pontificia Universidad Católica del Ecuador. Av. 12 de Octubre 1076 y Roca. Quito, Ecuador. Tel.: 59399 8412108. Correo: caramos@ puce.edu.ec
} 


\section{Introducción}

Existen tres niveles en donde se puede observar el control de la conducta en el ser humano. En primer lugar, se encuentra una forma de comportamiento totalmente automática, que estaría relacionada con sistemas cognitivos básicos. En un segundo nivel, existe un control del comportamiento semi-automático, en donde el ser humano actúa con patrones comportamentales habituales, previamente aprendidos. Y finalmente, en un tercer nivel, se encuentra el control del comportamiento totalmente consciente y regulado, que es controlado por las funciones ejecutivas (Norman \& Shallice, 1986; Slachevsky \& NúñezHuasaf, 2013).

Las funciones ejecutivas han sido definidas como un mecanismo de control que modula la operación de varios subprocesos cognitivos, controlando las dinámicas de la cognición humana, el control del pensamiento, el comportamiento y la afectividad; habilidades mentales que están en función del logro de una meta (Miyake et al., 2000). Tales procesos presentan un desarrollo prolongado, culminando en su maduración sobre el final de la adolescencia (Stelzer, Mazzoni, \& Cervigni, 2014).

Las funciones ejecutivas son un constructo complejo que, a su vez, hace referencia a procesos cognitivos y metacognitivos también complejos, con correlatos neuroanatómicos no definidos con exactitud, debido, entre otras cosas, a la falta de definiciones operacionales únicas de las funciones estudiadas, no obstante, son generalmente relacionados con el funcionamiento de los lóbulos frontales de la corteza cerebral (Tirapu-Ustárroz \& Luna-Lario, 2011).

En el proceso de identificación y definición de las funciones ejecutivas, desde la primera aproximación de Lezak (1982), se han ensayado distintas perspectivas teóricas, aunque básicamente, podríamos señalar la existencia de dos enfoques que permiten comprender a las funciones ejecutivas como producto de un conjunto de factores o bien, como un único factor central. En favor de la primera hipótesis, Gioia, Isquith, Retzlaff y Espy (2002) afirman que existen tres componentes primarios de las funciones ejecutivas, la regulación comportamental (conformada por la inhibición y monitorización personal), la metacognición (conformada por memoria de trabajo, iniciativa, planificación, organización de materiales y monitorización de tareas) y la regulación emocional (conformada por control emocional, y flexibilidad).

Para Brown (2008) las funciones ejecutivas se agrupan en las dimensiones: activación (conformado por organización, establecimiento de prioridades e iniciativa para trabajar), concentración (regulación voluntaria de la atención), esfuerzo (regulación del estado de alerta y velocidad del procesamiento de la información), emoción (regulación de la frustración y las emociones), memoria (memoria de trabajo y organización de la información adquirida) y acción (conformado por el automonitoreo y la autorregulación).

En cambio, en los modelos de funciones ejecutivas que responden a un sólo factor, se afirma que existe un único componente ejecutivo global, en donde actúan las funciones ejecutivas, como es el caso del modelo de Norman y Shallice (1986) que propone al sistema atencional supervisor o Barkley (1997) que propone al control inhibitorio como eje del funcionamiento ejecutivo.

Investigaciones previas han testeado los modelos explicativos de las funciones mediante la aplicación de métodos como el análisis factorial, en donde se han reportado resultados todavía no concluyentes, ya que no existe un acuerdo común sobre las diversas estructuras factoriales de las funciones ejecutivas y se han reportado estudios que proponen modelos de factores de primer orden de una función ejecutiva única o con varios factores metacognitivos, reguladores de la emoción y el comportamiento o modelos de segundo orden donde se consideran a funciones como la metacognición y la regulación del comportamiento en relación con un factor ejecutivo global (Arango, Puerta, \& Pineda, 2008; Egeland \& Fallmyr, 2010; Gioia et al., 2002; Ramírez, Pérez-Salas, \& Ortega, 2017; Roth, Lance, Isquith, Fischer, \& Giancola, 2013).

En la evaluación neuropsicológica de las funciones ejecutivas existen tres estrategias: (a) la aplicación de pruebas específicas, como son las tareas experimentales creadas para evaluar una función ejecutiva específica, como lo son las taras 
Go/No-Go, Stroop, Simon, entre otras (Ramos, 2017), (b) la aplicación de pruebas no específicas, que no han sido diseñadas para evaluar las funciones ejecutivas, sin embargo, son útiles en su valoración, como por ejemplo, las sub-pruebas de la escala de Wechsler (Ramírez \& Rosas, 2007) y (c) las pruebas de observación diferida, que consisten en las escalas de observación conductual, como el Behavior Rating Inventory of Executive Funcions (BRIEF; Gioia, Isquith, Guy, \& Kenworthy, 2000) o el cuestionario de evaluación de las funciones ejecutivas a través de la observación de la conducta: EFECO (GarcíaGómez, 2015).

En las dos primeras estrategias de evaluación de las funciones ejecutivas existe una limitación que debe ser resaltada, la cual consiste en la poca validez ecológica que tienen las tareas experimentales (Bakar, Taner, Soysal, Karakas, \& Turgay, 2011). Nos referimos por validez ecológica a la capacidad que tiene una prueba para hacer inferencias o predicciones fiables sobre el desempeño funcional del sujeto (BombínGonzález, y otros, 2014), por tanto, las pruebas ecológicamente válidas son aquellas que tienen características similares a las situaciones que encontraría un individuo en la vida real (Franzen \& Wilhelm, 1996).

En la evaluación neuropsicológica es frecuente encontrar que sujetos que presentan un desempeño conservado en tareas experimentales de la función ejecutiva (con poca validez ecológica) exhiben graves problemas de adaptación social y dificultades para tomar decisiones en la vida cotidiana (Damasio, 1994; Stuss \& Buckle, 1992). En tal sentido, se ha propuesto que, esta discrepancia entre el desempeño en el laboratorio y en la vida real, obedece a que el contexto de la evaluación neuropsicológica ofrece un entorno altamente estructurado y no refleja la capacidad real del paciente para hacer uso de sus recursos personales hacia la solución de los problemas reales. Adicionalmente, las pruebas clásicas de función ejecutiva evalúan únicamente ciertos aspectos de su complejidad, ignorando otros como los procesos metacognitivos en la vida real o la cognición social (Suchy, 2016).

El empleo de las escalas de observación conductual nace de la necesidad de superar la artificialidad de la evaluación en el laboratorio y valorar el funcionamiento ejecutivo del sujeto evaluando las actividades de su vida diaria (PérezSalas, Ramos, Oliva, \& Ortega, 2016). Por tal razón, es menester que el clínico cuente, dentro de sus reactivos para evaluar las funciones ejecutivas, con instrumentos de valoración conductual que respondan al contexto real en el cual se desenvuelve el individuo.

Una propuesta de evaluación ecológica es el cuestionario EFECO, que es un instrumento que permite evaluar las funciones ejecutivas: flexibilidad, organización de materiales, monitorización, control inhibitorio, control emocional, memoria de trabajo, iniciativa y planificación. Fue desarrollado para su reporte por parte de padres y/o profesores del paciente evaluado. Ha sido validado en España por GarcíaGómez (2015), donde se ha reportado que el instrumento cuenta con adecuadas propiedades psicométricas para su aplicación. Además, una de las bondades del cuestionario EFECO es su condición de uso libre, el cual se une a interesantes propuestas de uso abierto para evaluar las funciones ejecutivas, como lo es la plataforma PEBL (Mueller \& Piper, 2014) que dispone de diversos experimentos para evaluar estas funciones neuropsicológicas.

Por tanto, es indispensable realizar una investigación que permita valorar las propiedades psicométricas del cuestionario en diversos entornos donde no se lo ha realizado previamente y poder beneficiar con un reactivo de uso libre para investigadores interesados en el estudio de las funciones ejecutivas. En tal contexto, surge la necesidad de adaptar el cuestionario EFECO en una versión de auto-reporte, para que pueda ser aplicado en futuros estudios con estudiantes secundarios y universitarios. Además, es importante mencionar que, actualmente existe un vacío dentro de la línea de investigación de funciones ejecutivas en Latinoamérica, caracterizado por la ausencia de instrumentos de evaluación con validez ecológica de las funciones ejecutivas en formato de auto-reporte para población adolescente y juvenil.

El aporte de este estudio a la línea de investigación de las funciones ejecutivas radica en los siguientes aspectos, primero, existe una deficiencia de estudios psicométricos de 
instrumentos para valorar funciones ejecutivas en el contexto de habla hispana, además, este estudio aporta en un segmento de la investigación de funciones ejecutivas todavía no resuelto, que es el desarrollo de reactivos para su evaluación y diagnóstico; segundo, el instrumento EFECO ha sido desarrollado en formato de reporte por padres y maestros, sin embargo, no ha sido estudiada una versión de auto-reporte, y tercero, el proponer un instrumento novedoso que aporte al clínico en la evaluación de las funciones ejecutivas.

\section{Escenario de la investigación}

Esta investigación es realizada en Ecuador, un país de Latinoamérica con más de 16 millones de habitantes, que tiene un sistema económico capitalista, utiliza el dólar de los Estados Unidos de América como moneda oficial, la mayoría de su población es de religión Católica y su sistema educativo es similar al ofertado al de otros países de la región y otros contextos (Cruz-Cárdena, Arévalo-Chávez, \& Guadalupe, 2018). Estas características sociales, educativas y económicas de Ecuador, hacen que los resultados descritos a continuación, podrían ser interpretados para comprender el rol de las funciones ejecutivas en contextos que compartan características similares al del país.

\section{Método}

\section{Participantes}

La muestra estuvo conformada por 250 estudiantes secundarios del sistema educativo de Ecuador. El muestreo fue de tipo probabilístico de las 6 instituciones con mayor número de estudiantes de Quito, Ecuador. En cuanto al género, 119 participantes pertenecieron al sexo masculino (47.6\%) y 131 al femenino (52.4\%). El rango etario estuvo comprendido entre 13 y 18 años de edad $\left(M_{\text {edad }}=16.23, D E=1.73\right)$. El nivel educativo de los participantes estuvo entre décimo de educación general básica y tercero de bachillerato. El nivel de ingreso económico del hogar, características educativas de padres y contexto de vivienda de los participantes, son características de la clase socioeconómica media. Se incluyó de manera aleatoria a todos los participantes seleccionados mediante un cálculo estadístico, con lo cual se aportó validez externa al estudio. En la investigación los participantes no presentaban discapacidad alguna, trastorno neurológico o trastorno psicológico diagnosticado previamente.

\section{Análisis de datos}

En el paquete SPSS versión 21 se realizaron los procesos estadísticos descriptivos de tendencia central y dispersión, Alfa de Cronbach para analizar la consistencia interna de la escala, correlación de Pearson para analizar la relación entre los diferentes componentes de las funciones ejecutivas y $t$ de Student para analizar la validez discriminante del instrumento. En el paquete AMOS versión 23 se realizó un análisis factorial confirmatorio para valorar la validez de constructo.

\section{Diseño de investigación}

Se trata de un estudio de tipo cuantitativo, no experimental, temporalidad transversal, que busca la validación de un reactivo psicométrico en un contexto no aplicado previamente. Es una investigación instrumental, destinada a analizar las propiedades psicométricas del cuestionario EFECO y evaluar su estructura factorial latente (Ato, López, \& Benavente, 2013).

\section{Adaptación del instrumento EFECO}

Como se lo mencionó, el instrumento para evaluar las funciones ejecutivas EFECO fue desarrollado en España por García-Gómez (2015) en una versión de reporte conductual por parte de padres y profesores de estudiantes de nivel básico. Se compone de 67 ítems que se valoran en una escala tipo Likert de cero puntos cuando la respuesta es nunca, un punto cuando es a veces, dos puntos cuando se responde con frecuencia y tres puntos cuando la respuesta es con mucha frecuencia.

En cuanto a parámetros de consistencia interna de la escala original EFECO, para su aplicación mediante el reporte de padres y profesores, se ha encontrado que su coeficiente de Alfa de Cronbach es $\alpha=.96$ y en el análisis de dos mitades de Guttman .94 (García-Gómez, 2015).

El primer paso que se siguió en la adaptación del cuestionario, fue la contextualización lingüística de cada ítem para proponerlo en auto- 
reporte. Posteriormente, se solicitó el juicio experto de tres investigadores con amplia experiencia en funciones ejecutivas. Luego de insertar sugerencias en relación al contenido de los ítems, se procedió a ejecutar un estudio piloto con un grupo de estudiantes que no participaron en la investigación. En esta fase se constató que el instrumento se encontrara en un adecuado estado para su utilización, para posteriormente ser aplicado en grupos de alrededor 10 participantes.

En todo momento de la investigación se respetó los estándares éticos de investigación con seres humanos declarados en Helsinki (Nathanson, 2013; Williams, 2008). Se solicitó la firma de consentimiento y asentimiento de participación, en todo momento se mantuvo absoluta confidencialidad y se protegió la integridad física y psicológica de los participantes.

\section{Resultados}

En los descriptivos de cada escala se encontró una media $(M)$ y desviación estándar $(D E)$ de: inhibición $M=8.33 \quad(D E=4.53), \quad$ flexibilidad $M=5.15$ ( $D E=2.82$ ), control emocional $M=7.82$ ( $D E=4.79$ ), organización de materiales $M=6.29$ ( $D E=3.96$ ), monitorización $M=5.44$ ( $D E=3.40)$, iniciativa $M=7.25(D E=4.13)$, memoria de trabajo $M=8.09 \quad(D E=4.59) \quad$ y planificación $\quad M=6.28$ $(D E=3.36)$.

Para evaluar la consistencia interna del instrumento se realizó un análisis basado en el procedimiento de Alfa de Cronbach, en donde se encontró que el total de la escala presenta un coeficiente $\alpha=.95$, lo cual permite afirmar que el instrumento cumple con un parámetro adecuado de confiabilidad.

En cada escala del cuestionario se obtuvieron coeficientes de alfa de Cronbach aceptables: inhibición $\alpha=.76$, flexibilidad $\alpha=.64$, control emocional $\alpha=.83$, planificación $\alpha=.73$, organización de materiales $\alpha=.78$, monitorización $\alpha=.72$, iniciativa $\alpha=.77$ y memoria de trabajo $\alpha=.82$.

En el análisis de correlación de los ítems con el total de la escala, se encontró que correlacionan entre .30 y .60 , lo cual sugiere que los ítems se encuentran asociados entre sí y aportan de manera adecuada en la valoración de las funciones ejecutivas. Únicamente los ítems 43 y 53 presentan una correlación inferior, sin embargo, se observa que eliminarlos no mejora significativamente el coeficiente de consistencia interna de la escala, por lo que, se decidió conservarlos dentro del reactivo. En la Tabla 1 se observa la correlación de cada ítem con el total de la escala y el alfa si se eliminara dicho ítem.

En la conformación de las diferentes funciones ejecutivas se siguió la configuración propuesta por García-Gómez (2015), en donde se considera que cada función ejecutiva se compone por los siguientes ítems. Monitorización: 2, 6, 11, 12, 25, 29, 31, 35 y 43; inhibición: 3, 14, 15, 18, 21, 33, 34, 37, 42 y 46; flexibilidad cognitiva: 4, 23, 27, 32, 49 y 59; control emocional: 7, 19, 48, $50,55,63$ y 67; planificación: 22, 28, 39, 61, 62, 58 y 44; organización de materiales: 1, 9, 10, 26, $30,45,51$, y 60 ; iniciativa: $8,17,20,36,40,47$, $53,56,64$ y 65 ; y memoria de trabajo: $5,13,16$, $24,38,41,52,54,57$ y 66 . En la Tabla 2 se puede observar los coeficientes de correlación entre las diferentes sub-escalas del instrumento EFECO. Para analizar la validez de constructo de la escala se realizó un análisis factorial confirmatorio, donde se hipotetizaron tres modelos de funciones ejecutivas: (a) de un factor principal, que hemos denominado sistema supervisor ejecutivo global (ver Figura 1), (b) dos factores principales, denominadas como sistema supervisor de la conducta y sistema supervisor de la cognición (ver Figura 2) y (c) un tercer modelo con tres factores, sistema supervisor de la emoción, sistema supervisor de la cognición y sistema supervisor de la conducta (ver Figura 3). En la Tabla 3 se presentan los parámetros de bondad de ajuste de cada uno de los modelos.

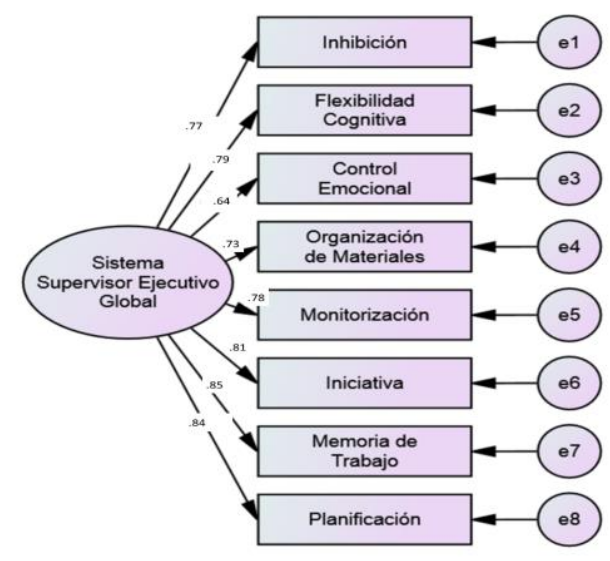

Figura 1. Modelo de la función ejecutiva con un factor principal de la función ejecutiva 
Tabla 1. Consistencia interna del cuestionario EFECO

\begin{tabular}{|c|c|c|}
\hline İtems & $\mathrm{r}_{\mathrm{jx}}$ & $\alpha-\mathrm{j}$ \\
\hline 1. Tengo dificultades para recoger y dejar ordenadas mis cosas cuando se me pide que lo haga. & .43 & .95 \\
\hline 2. Necesito tener alguien cerca para que me ayude a terminar una tarea cuando es muy larga. & .35 & .95 \\
\hline 3. Actúo sin pensar, haciendo lo primero que pasa por mi mente. & .35 & .95 \\
\hline 4. Tengo dificultad para admitir mis errores. & .41 & .95 \\
\hline 5. Me cuesta atender a las instrucciones que se me indican. & .50 & .95 \\
\hline 6. Cometo errores por descuido. & .38 & .95 \\
\hline 7. Me enfado por cosas insignificantes. & .52 & .95 \\
\hline 8. Tengo dificultades para tomar decisiones en forma independiente. & .49 & .95 \\
\hline 9. Dejo tiradas mis cosas por todas partes. & .34 & .95 \\
\hline 10. Tengo dificultades para encontrar rápidamente mis materiales al buscarlos en mi cuarto o escritorio. & .37 & .95 \\
\hline 11. Me cuesta realizar las tareas sin ayuda de los demás. & .41 & .95 \\
\hline 12. Soy lento/a en la realización de mis tareas educativas y del hogar. & .50 & .95 \\
\hline 13. Me cuesta concentrarme. & .56 & .95 \\
\hline 14. Tengo dificultades para esperar tranquilamente a que llegue mi turno. & .40 & .95 \\
\hline 15. Estoy moviéndome, no puedo estar quieto/a. & .43 & .95 \\
\hline 16. Hago mal mis tareas porque no sigo las instrucciones que se me dan. & .45 & .95 \\
\hline 17. Me cuesta hacer buenas propuestas para resolver problemas. & .45 & .95 \\
\hline 18. Tengo dificultad para escuchar atentamente. & .51 & .95 \\
\hline 19. Cuando me enfado tengo dificultad para calmarme con facilidad. & .46 & .95 \\
\hline 20. Parece que tengo ganas de hacer cosas, pero enseguida me olvido de ellas. & .56 & .95 \\
\hline 21. Interfiero o interrumpo las actividades de los demás. & .43 & .95 \\
\hline 22. Me resulta difícil pensar o planificar las cosas con antelación. & .44 & .95 \\
\hline 23. Me cuesta cambiar de una tarea a otra. & .40 & .95 \\
\hline 24. Me cuesta mantener la atención en una actividad. & .57 & .95 \\
\hline 25. Necesito de alguien que me supervise para realizar mis trabajos. & .54 & .95 \\
\hline 26. Tengo dificultades para cuidar mis pertenencias. & .51 & .95 \\
\hline 27. Me perturban los cambios de planes. & .46 & .95 \\
\hline 28. Hago mis tareas de forma apresurada. & .42 & .95 \\
\hline 29. Tengo dificultad para hacer todos mis deberes sin detenerme. & .42 & .95 \\
\hline 30. Me cuesta trabajo encontrar mis cosas cuando las necesito. & .46 & .95 \\
\hline 31. Me resulta difícil comportarme adecuadamente en las reuniones sociales. & .32 & .95 \\
\hline 32. Me resulta difícil dejar de hacer algo cuando se me pide que no lo haga más. & .55 & .95 \\
\hline 33. Interrumpo a los demás cuando están hablando. & .41 & .95 \\
\hline 34. Me cuesta anticipar las consecuencias de mis actos. & .44 & .95 \\
\hline 35. Necesito de la ayuda de un adulto para terminar las tareas. & .43 & .95 \\
\hline 36. Necesito que me animen constantemente para comenzar a hacer mis tareas educativas y del hogar. & .53 & .95 \\
\hline 37. Protesto cuando no se me deja hacer lo que quiero. & .54 & .95 \\
\hline 38. Me resulta difícil concentrarme en el desarrollo de todo tipo de juegos (p. ej. juegos de mesa). & .39 & .95 \\
\hline 39. Olvido revisar las tareas después de terminarlas. & .55 & .95 \\
\hline 40. Tengo dificultades para tomar decisiones, incluso ante las cosas más sencillas. & .62 & .95 \\
\hline 41. Me resulta difícil concentrarme en algo. & .56 & .95 \\
\hline 42. Puedo llegar a decir cosas inadecuadas a otras personas. & .50 & .95 \\
\hline 43.Tengo dificultades para realizar apropiadamente actividades o tareas que tienen más de un paso. & .08 & .95 \\
\hline 44. Me es difícil regresar a una tarea después de tomar un descanso & .41 & .95 \\
\hline 45. Olvido revisar mi cartera o mochila antes de ir a la Universidad/Colegio & .44 & .95 \\
\hline 46. Me levanto de la silla o abandono mi lugar cuando no debo hacerlo & .36 & .95 \\
\hline 47. Necesito que se me diga que comience una tarea, aunque tenga ganas de hacerla & .47 & .95 \\
\hline 48. Me altero o pierdo el control cuando se extravía algo & .51 & .95 \\
\hline $\begin{array}{l}\text { 49. Tengo dificultades para adaptarme a los cambios de mis rutinas, a nuevos profesores o a cambios en los } \\
\text { planes familiares }\end{array}$ & .46 & .95 \\
\hline 50. Me decepciono fácilmente & .43 & .95 \\
\hline 51. Parece que voy dejando todo desordenado por donde paso & .60 & .95 \\
\hline 52. Me distraigo fácilmente & .58 & .95 \\
\hline 53. Debo esforzarme incluso en las asignaturas que me gustan & .16 & .95 \\
\hline 54. Olvido llevar a casa las tareas, avisos o asignaciones educativas & .52 & .95 \\
\hline 55. Me molesto fácilmente & .44 & .95 \\
\hline 56. Me cuesta estar dispuesto/a a iniciar las tareas con sólo proponérmelas & .50 & .95 \\
\hline 57. Tengo problemas para concentrarme en la realización de tareas educativas y del hogar & .56 & .95 \\
\hline 58. Me quedo en los detalles de la tarea y pierdo el objetivo principal & .58 & .95 \\
\hline 59. Me resisto a resolver de forma diferente tareas educativas, juegos con amigos, tareas del hogar, etc. & .51 & .95 \\
\hline 60. Mi desorden tiene que ser recogido por otros & .55 & .95 \\
\hline 61. Tengo dificultad para terminar mis deberes a tiempo & .60 & .95 \\
\hline 62. Me cuesta tener buenos hábitos de estudio & .52 & .95 \\
\hline 63. Tengo cambios de ánimo de forma sorpresiva (triste, alegre, miedoso, sorprendido) & .46 & .95 \\
\hline 64. Me cuesta tener iniciativa para comenzar actividades, juegos o tareas educativas & .48 & .95 \\
\hline 65. Me cuesta tener muchas ideas & .39 & .95 \\
\hline 66. Me olvido de las cosas & .54 & .95 \\
\hline 67. Pierdo el control de mis emociones (hago rabietas) & .57 & .95 \\
\hline
\end{tabular}

Nota. $\mathrm{r}_{\mathrm{j} \mathrm{x}}$ Correlación ítem total escala, $\alpha$-j alfa si se elimina el ítem. 
Tabla 2. Correlación entre las sub-escalas del cuestionario EFECO

\begin{tabular}{lcccccccc}
\hline & M & IH & FX & CE & OM & IV & MT & PL \\
\hline M & - & & & & & & & \\
IH & $.51^{*}$ & - & & & & & & \\
FX & $.56^{*}$ & $.67^{*}$ & - & & & & & \\
CE & $.40^{*}$ & $.57^{*}$ & $.58^{*}$ & - & & & & \\
OM & $.50^{*}$ & $.58^{*}$ & $.55^{*}$ & $.40^{*}$ & - & & & \\
IV & $.58^{*}$ & $.61^{*}$ & $.65^{*}$ & $.58^{*}$ & $.57^{*}$ & - & & \\
MT & $.61^{*}$ & $.62^{*}$ & $.62^{*}$ & .49 & $.63^{*}$ & $.72^{*}$ & - & \\
PL & $.58^{*}$ & $.63^{*}$ & $.65^{*}$ & $.51^{*}$ & $.68^{*}$ & $.64^{*}$ & $.76^{*}$ & - \\
\hline Nota. M (monitorización), IH (inhibición), FX (flexibilidad), CE \\
(control emocional), OM (organización de materiales), IV \\
(iniciativa), MT (memoria de trabajo) y PL (planificación). \\
*. La correlación es significativa al nivel $p=.01$ (bilateral).
\end{tabular}

Tabla 3. Indicadores del análisis factorial confirmatorio

\begin{tabular}{|c|c|c|c|c|c|}
\hline Moc & $x^{2}$ & $G l$ & CFI & $\begin{array}{l}\text { RMSEA } \\
(95 \% \text { IC) }\end{array}$ & SRMR \\
\hline 1. $\mathrm{a}$ & $4.77 *$ & 20 & .96 & $.09(.07-.12)$ & \\
\hline 2. $b$ & $38.87 *$ & 19 & .98 & $.06(.03-.09)$ & .02 \\
\hline 3. $\mathrm{c}$ & $122.20^{*}$ & 24 & .93 & $.05(.10-.15)$ & .05 \\
\hline \multicolumn{6}{|c|}{$\begin{array}{l}\text { Nota. } \text { Gl=grados de libertad; RMSEA=error medio cuadrático de } \\
\text { aproximación; } 95 \% \mathrm{IC}=\text { intervalo de confianza; SRMR=residuo } \\
\text { estandarizado cuadrático medio; CFI=índice de ajuste } \\
\text { comparativo. a. Modelo de un factor: Sistema supervisor } \\
\text { ejecutivo global. b. Modelo de dos factores: Sistema supervisor } \\
\text { de la conducta y sistema supervisor de la cognición. c. Modelo } \\
\text { de tres factores: Sistema supervisor de la emoción, sistema } \\
\text { supervisor de la cognición y sistema supervisor de la conducta. } \\
* p<.01\end{array}$} \\
\hline
\end{tabular}

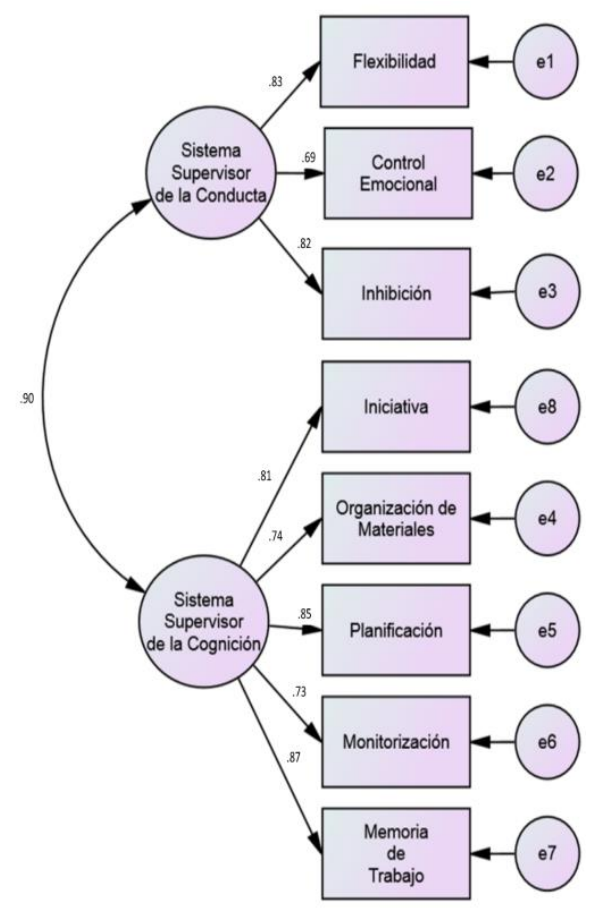

Figura 2. Modelo de la función ejecutiva con dos factores principales

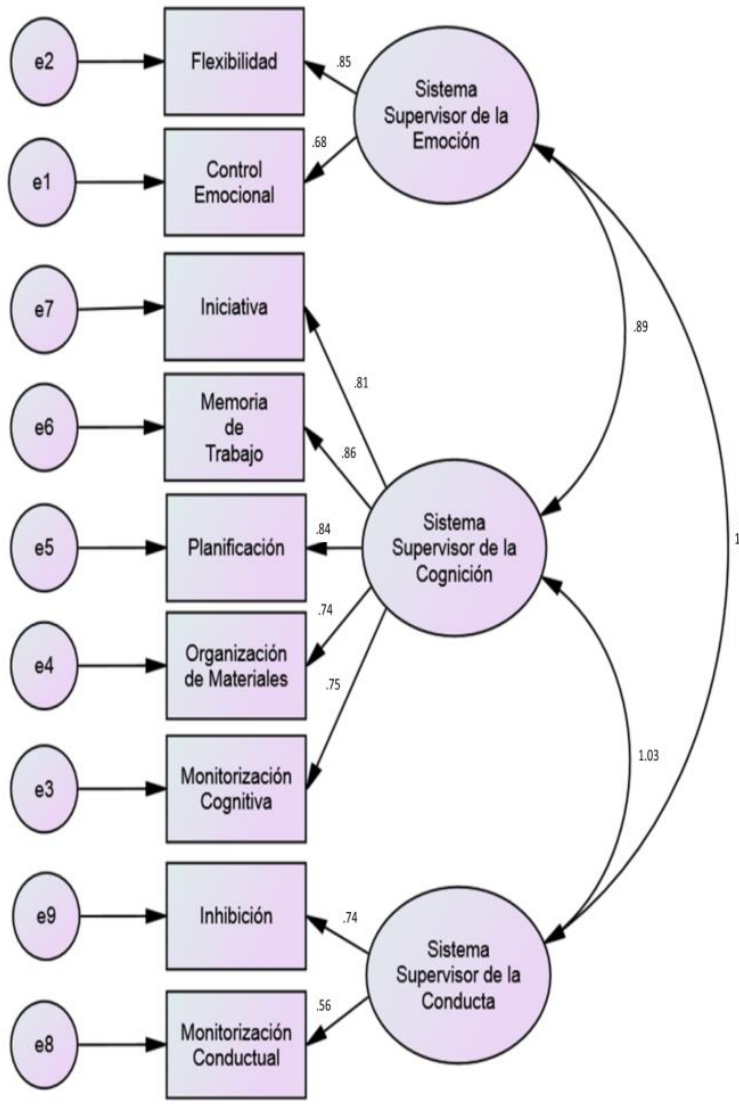

Figura 3. Modelo de la función ejecutiva con tres factores principales

Para analizar la capacidad discriminativa de la escala se la aplicó en dos grupos de estudiantes, (a) con alto rendimiento académico (99 estudiantes con una calificación promedio superior a 8 puntos sobre 10) y (b) bajo rendimiento académico (97 estudiantes con una calificación promedio inferior a 6 puntos sobre 10), y se los comparó en las ocho funciones ejecutivas que valora el EFECO (en la Tabla 4 se presentan los descriptivos). El parámetro cuantitativo del rendimiento académico se lo obtuvo mediante el reporte del estudiante. En este análisis se encontraron diferencias significativas en: monitorización $t_{(196)}=3.97, p=.001, d=.56$; flexibilidad cognitiva $t_{(196)}=3.47, p=.002, d=.49$; organización de materiales $t_{(196)}=3.39, p=.001$, $d=.48 ; \quad$ iniciativa $t_{(196)}=3.72, \quad p=.001, \quad d=.53$; memoria de trabajo $t_{(196)}=4.43, p=.001, d=.63 \mathrm{y}$ planificación $t_{(196)}=4.66, p=.001, d=.66$. En los puntajes encontrados, los estudiantes con bajo rendimiento académico presentaron mayores dificultades en sus funciones ejecutivas. Según la clasificación de Cohen (Cohen, 1988), el efecto 
para las diferencias en estas dimensiones es de magnitud media, situándose entre .48 y .66. En cambio, no se encontraron diferencias significativas en las funciones ejecutivas: inhibición $t_{(196)}=1.56, p=1.25, d=.22$ y control emocional $t_{(196)}=-.04, p=.88, d=.01$.

Tabla 4. Descriptivos de la comparación

\begin{tabular}{lcccc}
\hline $\begin{array}{l}\text { Funciones } \\
\text { Ejecutivas }\end{array}$ & Grupo & $\mathrm{N}$ & $\mathrm{M}$ & $\mathrm{SD}$ \\
\hline Inhibición & $\mathrm{G} 1$ & 97 & 9.14 & 3.83 \\
& $\mathrm{G} 2$ & 99 & 8.12 & 3.38 \\
Flexibilidad & $\mathrm{G} 1$ & 97 & 5.82 & 1.82 \\
& $\mathrm{G} 2$ & 99 & 4.61 & 1.70 \\
Control & $\mathrm{G} 1$ & 97 & 8.17 & 3.59 \\
emocional & $\mathrm{G} 2$ & 99 & 8.07 & 4.26 \\
Monitorización & $\mathrm{G} 1$ & 97 & 6.67 & 2.82 \\
Organización de & $\mathrm{G} 2$ & 99 & 4.51 & 2.06 \\
Matriales & $\mathrm{G} 1$ & 97 & 7.55 & 2.99 \\
Iniciativa & $\mathrm{G} 1$ & 99 & 5.50 & 2.84 \\
Memoria de & $\mathrm{G} 2$ & 99 & 8.49 & 3.79 \\
trabajo & $\mathrm{G} 1$ & 97 & 9.61 & 2.43 \\
Planificación & G2 & 99 & 6.85 & 2.71 \\
& G1 & 97 & 7.31 & 2.28 \\
& G2 & 99 & 5.47 & 2.21 \\
\hline
\end{tabular}

Nota. G1grupo de bajo rendimiento académico. G2 grupo de alto rendimiento académico.

\section{Discusión}

En el artículo se ha reportado un estudio que tuvo como objetivo adaptar el instrumento EFECO de evaluación de las funciones ejecutivas en un formato de auto-reporte.

Se realizaron varios análisis destinados a identificar la confiabilidad y validez del instrumento. En primer lugar, se realizó un análisis de Alfa de Cronbach, en donde se encontró que la escala total del instrumento y las sub-escalas presentan un coeficiente de consistencia interno adecuado. Estos resultados son concordantes con lo reportado por GarcíaGómez (2015) en su validación del instrumento EFECO reportado por padres y maestros en población española.

Nótese que, tanto el cuestionario en formato de reporte por los adultos responsables de niños, como en el formato de auto-reporte completado por adolescentes, se encuentra un parámetro de confiabilidad adecuado, lo que hace tener presente que el instrumento EFECO es útil en su aplicación con diferentes fuentes de información.
Prieto y Delgado (2010) señalan que cuando las puntuaciones vayan a emplearse para tomar decisiones que impliquen consecuencias relevantes para las personas (p. ej., aceptación o rechazo en una selección de personal, o para tomar decisiones sobre la inclusión en alguna categoría diagnóstica), se precisan coeficientes de fiabilidad muy altos (al menos de .90). Sin embargo, si se trata de describir las diferencias individuales a nivel de grupo, bastaría con alcanzar valores más modestos entre .70. Por lo cual, una de las principales implicaciones del estudio es el aporte que se realiza para que el clínico, interesado en el estudio de las funciones ejecutivas, cuente con un instrumento de cribado dentro de toda la complejidad que implica la evaluación de estas funciones de alto orden.

Además, el parámetro de confiabilidad es similar al reportado en el análisis de otras escalas de reporte conductual de las funciones ejecutivas, como por ejemplo el BRIEF, donde se ha reportado coeficientes de confiabilidad similares a los encontrados en el presente estudio (García, González-Pienda, Rodríguez, Álvarez, \& Álvarez, 2014; Pérez-Salas et al., 2016).

En el análisis de aporte de cada ítem al total de la escala, se encontró que todos los ítems, a excepción de los ítems 53 y 43, presentan un elevado nivel de correlación con el resto de la escala, sin embargo, el eliminar estos dos ítems, no se mejoraría el coeficiente de Alfa de Cronbach, por lo que, se ha decidido conservarlos en el instrumento, además, aportan de manera significativa desde una perspectiva clínica y teórica al estudio de la función ejecutiva.

En la correlación entre las diferentes funciones ejecutivas de la escala EFECO se encontró que todas son significativas en una magnitud importante, lo cual confirma la suposición de Gioia et al. (2002), quienes afirman que las funciones ejecutivas podrían estudiarse como componentes independientes desde un punto de vista teórico y clínico, no obstante, estarían altamente interrelacionadas, ya que pertenecen a un componente ejecutivo global (Pérez-Salas et al., 2016).

En el análisis de validez de constructo se encontró que, el modelo de dos factores de la función ejecutiva presenta mejores índices de ajuste, ya que los parámetros del CFI son mayores 
a .90, SRMR menor a .05 y el parámetro de RMSEA se encuentra inferior a .07 ( Hu \& Bentler, 1995; Steiger, 2007). Este resultado tiene concordancia con el modelo propuesto por Gioia et al. (2000) y Ramírez et al., (2017), que afirma que las funciones ejecutivas se organizan en dos factores principales: la regulación comportamental y la metacognición que, en nuestro caso, los identificamos como sistema supervisor de la conducta y sistema supervisor de la cognición.

Por otro lado, se puso a prueba un modelo que consideró nueve funciones ejecutivas, dividiendo a monitorización en cognitiva y conductual, teniendo como guía al trabajo publicado por Gioia et al. (2002), sin embargo, los índices de ajuste del presente trabajo sugieren que las funciones ejecutivas se organizarían en las dos dimensiones hipotetizadas, a pesar de que, los índices encontrados para nuestro modelo de tres factores son similares a lo reportado por Gioia et al. (2002), pero no mejores que el modelo de dos factores testeado.

Para analizar la capacidad discriminativa del EFECO se valoró el funcionamiento ejecutivo en dos grupos de estudiantes: con alto y bajo rendimiento académico. En esta comparación se encontró que, los estudiantes con alto rendimiento académico presentan un mejor desempeño en las funciones ejecutivas, a diferencia de los estudiantes con bajo rendimiento académico, quienes presentan mayores dificultades en su funcionamiento ejecutivo.

Este hallazgo tiene relación con lo afirmado por Mulder y Cragg (2014), Jacob y Parkinson (2015) y Reyes, Barreyro y Injoque-Ricle (2015), quienes aseveran que las funciones ejecutivas tienen una relación directa con el rendimiento académico, ya que estas funciones cognitivas de alto nivel, serían factores que pueden determinar el éxito o fracaso de un estudiante en el contexto educativo. En este sentido, Meltzer (2010) puntualiza que el éxito académico de los alumnos depende de sus habilidades para planificar y priorizar el tiempo, para organizar los materiales y la información disponible, para separar las ideas fundamentales de las accesorias, para cambiar de rumbo de forma flexible en una actividad cuando sea requerido, controlar o monitorizar el desarrollo de las actividades y reflexionar sobre el trabajo realizado.
Como limitaciones del presente estudio se deben mencionar las siguientes: en primer lugar, la muestra pertenece a una ciudad determinada de Latinoamérica, en segundo lugar, se debe tener presente el carácter subjetivo implícito en la aplicación de la prueba de auto-reporte, que puede estar sesgado por la propia percepción del individuo sobre su situación conductual; y en tercer lugar, no se contó con una medida de comparación, que permita realizar el análisis de validez concurrente, sin embargo, estos tres aspectos no invalidan al presente estudio, ya que al no tener un instrumento previo con las características de la escala EFECO, este estudio pionero brinda importante información psicométrica para el contexto clínico y científico interesado en el estudio de las funciones ejecutivas.

La línea de investigación futura que se desprende de este estudio invita a investigar la validez convergente del cuestionario EFECO con tareas experimentales de evaluación de la función ejecutiva y analizar la correlación entre las funciones ejecutivas con variables implicadas en el rendimiento académico de estudiantes del nivel académico descritos en el estudio.

\section{Financiamiento}

Esta investigación no recibió financiamiento externo.

\section{Aprobación Ética}

Esta investigación fue aprobada por el Comité de Ética de la Universidad Tecnológica Indoamérica (Ecuador). Todos los procedimientos que se aplicaron se encontraron en concordancia con la Declaración de Helsinki, en donde se precauteló la integridad física y psíquica de los participantes. El consentimiento informado fue firmado por los participantes una vez que se les explicó los objetivos de la investigación y previamente a la aplicación de la escala EFECO.

\section{Conflicto de Intereses}

Los autores no presentan ningún tipo de conflicto de interés. 


\section{Referencias}

Arango, O., Puerta, I., \& Pineda, D. (2008). Estructura factorial de la función ejecutiva desde el dominio conductual. Diversitas, 4(1), 63-77.

Ato, M., López, J., \& Benavente, A. (2013). Un sistema de clasificación de los diseños de investigación en psicología. Anales de Psicología, 29(3), 1038-1059. doi:10.6018/analesps.29.3.178511

Bakar, E., Taner, Y., Soysal, A., Karakas, S., \& Turgay, A. (2011). Behavioral rating inventory and laboratory tests measures different aspects of executive functioning in boys: A validity study. Bulletin of Clinical Psychopharmacology, 21(4), 302-316. doi:10.5455/bcp.20111004014003

Barkley, R. (1997). Behavioral inhibition, sustained attention, and executive functions: Constructing a unifying theory of ADHD. Psychological Bulletin, 121(1), 65-94. doi:10.1037/0033-2909.121.1.65

Bombín-González, I., Cifuentes-Rodríguez, A., Climent-Martínez, G., Luna-Lario, P., CardasIbáñez, J., Tirapu-Ustárroz, J., \& Díaz-Orueta, U. (2014). Validez ecológica y entornos multitarea en la evaluación de las funciones ejecutivas. Revista de Neurología, 59(2), 7787.

Brown, T. (2008). ADD/ADHD and impaired executive function in clinical practice. Current Psychiatry Reports, 10 (5), 407-411. doi:10.1007/s12618-009-0006-3

Cohen, J. (1988). Statistical power analysis for the behavioral sciences. New Jersey: Lawrence Erlbaum.

Cruz-Cárdenas, J., Arévalo-Chávez, P., \& Guadalupe, J. (2018). Consumer expenditures on clothing and footwear: A mixed methods study. Journal of Fashion Marketing and Management, $\quad 22(1), \quad$ 99-113. doi:10.1108/JFMM-12-2016-0121

Egeland, J., \& Fallmyr, Ø. (2010). Confirmatory factor anlysis of the Behavior Rating Inventory of Executive Function (BRIEF): Support for a distinction between emotional and behavioral regulation. Child Neuropsychology, 16(4), 326-377. doi:10.1080/09297041003601462
Damasio, A. (1994). El error de Descartes. Santiago de Chile: Editorial Andrés Bello.

Franzen, M., \& Wilhelm, K. (1996). Conceptual foundations of ecological validity in neuropsychological assessment. En C. Sbordone, \& J. Long, Ecological Validity of Neuropsychological Testing (págs. 91-112). Delray Beach: Lucie Press.

García, T., González-Pienda, J., Rodríguez, C., Álvarez, D., \& Álvarez, L. (2014). Psychometric characteristics of the BRIEF scale for the assessment of executive functions in Spanish clinical population. $\begin{array}{llll}\text { Psicothema, } & 26 & \text { (1), 47-52. }\end{array}$ doi:10.7334/psicothema2013.149

García-Gómez, A. (2015). Desarrollo y validación de un cuestionario de observación para la evaluación de las funciones ejecutivas en la infancia. Revista Intercontinental de Psicología y Educación 17 (1), 141-162.

Gioia, G., Isquith, P., Guy, S., \& Kenworthy, L. (2000). Behavior Rating Inventory of Executive Function. Lutz, Florida: Psychological Assessment Resources.

Gioia, G., Isquith, P., Retzlaff, P., \& Espy, K. (2002). Confirmatory factor analysis of the Behavior Rating Inventory of Executive Function (BRIEF) in a Clinical Sample. Child Neuropsychology, 8 (4), 249-257. doi:10.1177/0031512516650441

Hu, L., \& Bentler, P. (1995). The bi-factor method. Psychometrika, 47, 41-54.

Jacob, R., \& Parkinson, J. (2015). The potential for school-based interventions that target executive function to imrpove academic achievement: A review. Review of Educational Researh, 85 (4), 512-552. doi:10.3102/0034654314561338

Lezak, M. (1982). The problem of Assesing Executive Functions. International Journal Psychology, 17, 281-297. doi:10.1080/00207598208247445

Meltzer, L. (2010). Promoting executive function in the classroom. New York: The Guilford Press.

Miyake, A., Friedman, N., Emerson, M., Witzki, A., Howerter, A., \& Wager, T. (2000). The unity and diversity of executive functions and their contributions to complex $\mathrm{x}$ 'frontal Lobe' tasks: A latent variable analysis. 
Cognitive Psychology, 41, 49-100. doi:10.1006/cogp.1999.0734

Mueller, S., \& Piper, B. (2014). The Psychology Experiment Building Language (PEBL) and PEBL Test Battery. Journal of Neuroscience Methods, 222, 250-259. doi:10.1016/j.jneumeth.2013.10.024

Mulder, H., \& Cragg, L. (2014). Executive functions and academic achievement: Current research and futures directions. Infant and Child Development, 23(1), 1-3. doi:10.1002/icd.1836

Nathanson, V. (2013). Revising the declaration of Helsinki. BMJ, 346, 1-2. doi:10.1136/bmj.f2837.

Norman, D., \& Shallice, T. (1986). Attention to action: Willed and automatic control of behavior. En R. Davidson, G. Schwartz, \& D. Shapiro, Consciousness and self-regulation (págs. 1-18). New York: Editorial Plenumm Press.

Pérez-Salas, C., Ramos, C., Oliva, K., \& Ortega, A. (2016). Bifactor modeling of the Behavior Rating Inventory of Executive Function (BRIEF) in a Chilean Sample. Perceptual and Motor Skills, 122 (3), 757-776. doi:10.1177/0031512516650441

Prieto, G., \& Delgado, A. (2010). Fiabilidad y Validez. Papeles del Psicólogo, 31(1), 67-74.

Ramírez, P., Pérez-Salas, C., \& Ortega, A. (2017). Análisis de la estructura jerárquica del inventario de conductas de la función ejecutiva en una muestra de estudiantes universitarios. Revista Iberoamericana de Diagnóstico y Evaluación - e Avaliação Psicológica, 45(3), 175-188. doi:10.21865/RIDEP45.3.14

Ramírez, V., \& Rosas, R. (2007). Test de inteligencia para niños de Wechsler. Santiago de Chile: Ediciones Universidad Católica.

Ramos-Galarza, C. (2017). Adaptación del Test Stroop Victoria en Estudiantes Ecuatorianos. Revista Iberoamericana de Diagnóstico y Evaluación - e Avaliação Psicológica, 44(2), 57-64. doi.org/10.21865/RIDEP44.2.05.

Reyes, S., Barreyro, J., \& Injoque-Ricle, I. (2015). El rol de la función ejecutiva en el rendimiento académico en niños de 9 años. Revista Neuropsicología Latinoamericana, 7 (2), 42-72. doi:10.5579/rnl.2015.0229
Roth, R., Lance, C. E., Isquith, P. K., Fischer, A. S., \& Giancola, P. R. (2013). Confirmatory factor analysis of the behavior rating inventory of executive function-adult version in healthy adults and application to attention deficit/hyperactivity disorder. Archives of clinical neuropsychology, 28(5), 225-234. doi:10.1093/arclin/act031

Slachevsky, A., \& Núñez-Huasaf, J. (2013). Procesos Ejecutivos y Adaptación del Comportamiento. En J. Lavados, \& A. Slachevsky, Neuropsicología de los Procesos Mentales (págs. 189-209). Santiago, Chile: Editorial Mediterráneo.

Steiger, J. (2007). Understanding the limitations of global fit assessment in structural equation modeling. Personality and Individual Differences, 42(5), 893-898. doi:10.1016/j.paid.2006.09.017

Stelzer, F., Mazzoni, C., \& Cervigni, M. (2014). Cognitive models of executive functions development. Metodological limitations and challenges. Anales de Psicología, 30(1) , 329336. doi:10.6018/analesps.30.1.139251

Stuss, D., \& Buckle, L. (1992). Traumatic brain injury: Neuropsychological deficits and evaluations at different stages of recovry and different pathologic subtypes. Journal of Head Trauma Rehabilitation, 7(2), 40-49.

Suchy, Y. (2016). Executive functions: A Comprehensive guide for clinical practice. New York: Oxford University Press.

Tirapu-Ustárroz, J., \& Luna-Lario, P. (2011). Neuropsicología de las funciones ejecutivas. En J. Tirapu-Ustárroz, M. Ríos-Lago, \& F. Maestú, Manual de Neuropsicología Segunda Edición (págs. 221-259). Barcelona, España: Viguera Editores.

Williams, J. (2008). Revising the declaration oh Helsinki. World medical journal. 54 (4), 120122. 